\title{
FILOSOFÍA CON NIÑOS EN TUCUMÁN
}

\author{
Julieta Teitelbaum $\underline{(1)}$
}

RESUMEN: Desde una perspectiva que concibe a la Filosofía no como un saber conceptual y abstracto, sino como una actitud fundamentalmente interrogativa y vital; y desde una mirada que advierte el carácter lúdico y cuestionador que prevalece en la infancia, podemos hablar de la posibilidad de hacer "Filosofía con niños". Es decir, podemos pensar en un espacio compartido y vivido con los niños, donde se lleven a cabo experiencias que estimulen la actitud filosófica. En diversos lugares esta posibilidad ya tiene una gran trayectoria, en otros recién está comenzando. Tal es el caso de la Provincia de Tucumán, a la cual se hará referencia en este trabajo.

\section{FILOSOFÍA: UNA CUESTIÓN DE ACTITUD}

La filosofía ha sido considerada tradicionalmente como un conjunto de conocimientos abstractos, de difícil acceso y alejados de la vida cotidiana. Se creía que para ingresar en el complejo mundo de las ideas filosóficas, era necesario, poder hablar de grandes y estáticos conceptos como el Bien, la Verdad o la Belleza. Y dado que la filosofía era un asunto elevado no estaba destinado para cualquiera: las mujeres, los niños, los hombres comunes, no eran bienvenidos a la academia filosófica. En la misma categoría que las cosas, pertenecían a un mundo inferior y sensible del cual no quedaba más remedio que huir si se quería contemplar la verdadera esencia de lo real. Esta perspectiva eminentemente platónica, ha trazado fuertemente, no sólo una concepción acerca de la filosofía, sino también una manera de ser que incide, aún hoy, en Occidente. No es casual que la Carrera de Filosofía de la Universidad Nacional esté configurada de tal modo que en algún momento resulta inevitablemente absurda o vacía para el estudiante. En general, quien se inscribe en la Licenciatura o Profesorado de Filosofía, tiene al menos algún anhelo de encontrar un espacio de reflexión donde se discutan ideas, se formulen preguntas o se cuestionen supuestos; pero en su recorrido por la Facultad, el estudiante encuentra fundamentalmente la exigencia de aprender historia de la filosofía, de saber repetir el pensamiento de otros. Y sin embargo, no es en la demanda académica de leer los textos donde reside el sin sentido, pues esta tarea es altamente necesaria, sino en tener que postergar ese deseado espacio de reflexión para más adelante, cuando se suponga que ya se ha conseguido incorporar la biblioteca de la filosofía antigua, medieval y moderna. Claro que, para cuando eso ocurra, la motivación y el entusiasmo primigenio se habrán convertido en una pretensión ridícula e infantil.

No resulta extraño, entonces, que en algún instante de la carrera surja la pregunta por cual sea el sentido de la filosofía. Y si bien esta pregunta puede generar angustia más que satisfacción, representa un corte en el camino que se venía 
siguiendo (literalmente hablando) hasta el momento, pues es a partir de la sensación de absurdo que comienzan a movilizarse ciertas ideas que se creían ya instaladas e incuestionables. ¿Es la filosofía un conjunto de conocimientos definidos por los grandes pensadores? ¿Es lo mismo leer textos filosóficos que hacer filosofía? ¿Es posible un espacio de reflexión y discusión de ideas? Estos y otros interrogantes iluminan un nuevo comienzo, recuperan un camino que ya se creía perdido. La filosofía es vista ahora como una actitud ante el mundo, actitud fundamentalmente interrogativa si acordamos que son las preguntas las que distinguen una mirada natural o corriente, acostumbrada a que las cosas sean como son, de una mirada filosófica que reclama algún tipo de explicación de "Por qué las cosas son como son".

La filosofía se vuelve de este modo más cercana, más cotidiana y visceral, apta para todo aquel que busca, que cuestiona, que desconfía de sus propios supuestos. Aparece como una actividad que como tal no está predeterminada, sino abierta a lo que pueda suceder cuando se interactúa con otros y de alguna manera con uno mismo; sus resultados son desconocidos, no sabemos de antemano a dónde vamos a llegar. "La práctica de la filosofía conlleva riesgos. No promete tesoros ciertos al final del camino. Es una forma de abrir el pensar a sí mismo y a lo nuevo, abrirlo a nuevas formas de pensar y a pensar de nuevo" (WAKSMAN, et alii, 2000, p. 70.)

La filosofía comienza a ser más que nada un modo de ver problemáticamente el mundo, una mirada incómoda, porque no se queda tranquila con aquello que percibe, sino que sospecha que eso que llamar mirar no es más que un punto de vista.

Si se entiende a la filosofía de este modo, la pretensión de alcanzar un saber conceptual se vuelve cuestionable y se adoptan nuevos presupuestos, entre los cuales el más importante parece ser el de incorporar a quienes habían quedado fuera de la posibilidad de pensar. Me ocuparé, en este trabajo, de un grupo en particular: los niños.

\section{LOS NIÑOS EN ACTITUD FILOSÓFICA}

La relación entre la filosofía y los niños resuena con mayor frecuencia en la actualidad. Han cambiado los criterios a partir de los cuales se definía la filosofía, podemos decir lo mismo respecto de la infancia. La posibilidad de una "filosofía para niños", mejor aún, de una "filosofía con niños" (como sostienen Walter Kohan y Vera Waksman) revela ciertas condiciones que la hacen posible. Me refiero que para poder hablar actualmente de la filosofía y los niños era necesario un cambio en el modo de entender la filosofía y también un cambio en la manera de mirar a los niños.

En general, resaltamos la infancia como una etapa de mayor espontaneidad y creatividad en contraste con la seriedad exigida en la adultez. Se reconoce que es en la niñez donde aparecen las grandes preguntas, el asombro ante lo desconocido, la necesidad de experimentar a través del cuerpo y los sentidos. Pero al mismo tiempo, no se advierte que todas estas cualidades se adormecen con el 
transcurso de los años o no se vislumbra el peligro que hay en ello. Es decir, por un lado celebramos el carácter lúdico y la búsqueda constante de los niños y por otro lado, no les damos la posibilidad de que desarrollen sus potencialidades. La escuela admite un espacio para el juego, el movimiento, las preguntas, la experimentación sólo en el Jardín de infantes o Nivel Inicial, no así en la educación posterior de los niños donde este espacio se ve frustrado o simplemente anulado. Desde primer grado, los pequeños estudiantes deben acostumbrarse a renunciar a lo que para ellos resulte interesante o entretenido a cambio de asumir obligaciones y responsabilidades. No es casual que a partir de aquí los niños tengan que realizar "deberes" o tareas. Se produce, de este modo, un cambio brusco entre los primeros años de educación escolar donde existe la oportunidad de interactuar con otros (compartiendo la mesa, los juegos, la merienda), de inventar e imaginar, de sentarse en círculo a escuchar un cuento, de moverse, de reírse; y la educación posterior que insiste en que se repriman estas actitudes. Se observa un corte, un paso del movimiento a la quietud (hay que permanecer sentados la mayor parte del tiempo), de las preguntas que hacen los niños a las preguntas que hacen los maestros, del entusiasmo al aburrimiento.

Pareciera que la actitud cuestionadora, atribuida a los niños no hace más que amenazar los cimientos de lo que está prolijamente construido. Cuando llegamos a ser lo que supuestamente soñábamos cuando éramos niños, miramos hacia atrás con cierta nostalgia, pero a la vez con cierta sensación de alivio dado que nos hemos liberado de la incomodidad que genera preguntar por qué la cosas son como son.

¿En qué sentido, entonces, valoramos la infancia? ¿En qué medida propiciamos una actitud creativa y crítica? si ser espontáneos y curiosos no nos resulta tan fascinante como decimos.

Ahora bien, a la par de una mirada evasiva de la niñez, que muestra sólo el rostro amable y claro de las cosas, surge una nueva posibilidad para recuperar lo que está oculto detrás de ese rostro, una nueva forma de concebir la infancia donde se pretende escuchar más que manipular con las palabras, dejarse llevar más que conducir, dejar ser a los niños más que a los adultos. Hablemos, entonces, de la posibilidad de hacer "filosofía con niños".

Como afirman Walter Kohan y Vera Waksman, "lo que se propone no es una filosofía para ellos, no es sólo poner a su alcance algo que antes era inaccesible. Filosofía con niños significa que la filosofía es algo que los niños pueden practicar y que ambos términos se modifican en su encuentro. No se trata de niños prodigio ni de filósofos precoces. Se trata de niños viviendo la experiencia de la filosofía" (WAKSMAN, et alii, 2000, p. 7).

Sabemos que el camino iniciado por Mattew Lipman a partir de la propuesta del Programa "Filosofía para niños" se ha extendido ampliamente por diversos países e Instituciones educativas. En algunos casos, el camino se ha enriquecido con nuevos aportes e interpretaciones y en otros, se ha implementado el programa sin demasiada revisión. La expresión "filosofía para niños" o más bien "filosofía con 
niños" puede pasar desapercibida en aquellos lugares donde se viene desarrollando hace tiempo algún tipo de experiencia formal o no formal, pero en otros ámbitos, estas expresiones producen perplejidad y cuestionamientos. "¿Qué es esto de filosofía con niños? ¿Cómo darles filosofía a los niños? ¿Para qué implementar la filosofía desde el Nivel Inicial? ¿No es absurdo que los niños hagan filosofía?". En este trabajo voy a referirme a estos espacios donde la relación entre la filosofía y los niños resuena lejana o es todavía incipiente. Voy a ocuparme en particular de abordar lo qué ocurre en la Provincia de Tucumán.

\section{LA EXPERIENCIA DE LA FILOSOFÍA EN TUCUMÁN}

El panorama puede resultarnos desalentador, si tenemos en cuenta que son solamente dos los lugares que se dedican a la "filosofía con niños". Sin embargo, la mayor trayectoria de algunas provincias o países pueden valer como experiencias que sirven para enriquecer el trabajo. Si el terreno no está todavía habitado, es claro que tenemos mucho por hacer, con la ventaja de poder abrir nuevos caminos. En la Ciudad de San Miguel de Tucumán, Capital de la provincia, existen dos escuelas de gestión privada que cuentan con una propuesta de "filosofía con niños", aunque con algunas diferencias relevantes. En la primera escuela a la que haremos referencia, la filosofía forma parte de la estructura curricular desde el Nivel Inicial (a partir de los 3 años) hasta 3ero del Polimodal (es decir hasta los 17 años). El perfil de la Institución se define acorde a la aplicación de la "Teoría de las Inteligencias Múltiples" propuesta por Howard Gardner, donde se considera fundamentalmente que no existe una única manera de ser inteligente, puesto que, cada persona posee una serie de habilidades o capacidades más desarrolladas que otras, vinculadas no sólo con el conocimiento lingüístico o matemático, sino también con el cuerpo, el espacio, la relación con los demás y con uno mismo. Se concede, desde esta perspectiva, una importancia central a la filosofía en tanto es capaz de estimular y desarrollar en los estudiantes las llamadas "habilidades de pensamiento": planteo de preguntas, formulación de hipótesis, búsqueda de conceptos, capacidad de análisis y de síntesis, observación, descripción, interpretación, etc. La filosofía es vista, no como un recorrido histórico de distintos autores o pensadores, sino como una experiencia que permite plantear problemas, proponer soluciones alternativas, formular preguntas. La intención no es que los niños repitan lo que dijeron Platón o Aristóteles, sino que a partir de su propia vivencia, de sus intereses e inquietudes más cercanas logren reflexionar, ir más allá de lo que parece incuestionable, establecido. Se apunta fundamentalmente a la adquisición de contenidos procedimentales y actitudinales para ir gradualmente trabajando con diversas temáticas y conceptos.

Hace cuatro años que se está llevando a cabo la experiencia de hacer filosofía con los niños y tuve la oportunidad de estar a cargo de la misma desde el año pasado.

Debo confesar que los niños ya estaban acostumbrados a que la filosofía forme parte de su vida escolar y quien estaba absolutamente sorprendida con dicha posibilidad era yo. Apenas ingresé al aula pude advertir que había algo que estaba claro para ellos: la filosofía implicaba sobre todo preguntar, -"la filosofía, señorita, es todo por qué, por qué, por qué.."- respondían conjuntamente, pero la filosofía 
era también una materia curricular, una serie de tareas que debían realizar obligatoriamente, y esto ya no resultaba tan encantador. Ante esta situación es inevitable cuestionarse si no resulta contradictorio querer propiciar un espacio de reflexión de manera obligatoria, si no resulta absurdo pensar sin que uno lo desee y peor aún, si no nos juega en contra una propuesta de este tipo hasta el punto que después se termine rechazando la filosofía porque al fin y al cabo sigue siendo una materia más de la escuela. Podemos decir que estos interrogantes no anularon la posibilidad de la filosofía con niños en la escuela, sino que sirvieron para delimitar los criterios con los cuales se iba a desarrollar. Uno de los más importantes es el que concierne a la evaluación, dado que es en este punto donde podemos encontrar mayor conflicto si pensamos en todas las implicancias negativas que conllevan los mecanismos de clasificación en la educación de los niños. Se discutieron, entonces, los presupuestos a partir de los cuales se evalúa a los estudiantes para llegar luego al acuerdo de que sean ellos mismos quienes delimiten qué debería ser evaluado, cómo y por qué. De esta manera, se superaría uno de los obstáculos que suele impedir que la filosofía resulte verdaderamente una experiencia movilizante.

La propuesta de hacer filosofía con niños no incluye en esta escuela, un programa curricular ni material bibliográfico definido, sino que es lo suficientemente flexible como para formular las actividades y recursos que se crean convenientes. En general, se ha recurrido a la literatura como uno de los principales disparadores: desde los cuentos clásicos hasta narraciones actuales, poesías, disparates, adivinanzas han sido un estímulo para la reflexión, y la discusión. Con los más pequeños, las canciones generaron mejores resultados como también la dramatización, los títeres, la plástica. En algunos casos se ha trabajado con las novelas filosóficas escritas por Lipman, Stella Accorinti, Gustavo Santiago. La manera de abordar el trabajo no se limitó a la lectura de textos, sino que además, se hicieron numerosas actividades lúdicas y ejercicios que ponían en juego diversos procedimientos, actitudes y temáticas. Se ha insistido fundamentalmente en la formulación de preguntas o problemas por parte de los niños, en la búsqueda de razones y argumentos para sostener una afirmación, en el reconocimiento de supuestos, en la clarificación de conceptos. Para citar algunos ejemplos, podemos mencionar: la elaboración por parte de los niños de su propio diccionario filosófico en el que anotaban las palabras que a ellos les parecían importantes para luego intentar definirlas, historias colectivas inventadas y grabadas por ellos, dramatizaciones sobre algunos cuentos trabajados, historietas y dibujos relativos a la mitología, numerosas discusiones acerca de la identidad, el tiempo, el origen del universo, el saber científico, los sueños, la amistad, la relación entre el hombre y las máquinas, el significado de ser niños y adultos, de ser mujeres y varones, las reglas y las normas, los valores, el destino, lo real y lo ficticio, etc.

Hasta aquí lo que concierne a la manera de hacer filosofía con niños en una de la escuelas de Tucumán. Pasemos, ahora al segundo caso.

Se trata de una escuela también privada, donde la filosofía acaba de ser incorporada en los planes de estudio. Dado que la institución tiene una tendencia hacia la economía y la contabilidad, las materias humanísticas no ejercen un peso 
muy decisivo. Este año se incluyó "lógica" en 3ero del Polimodal y se propuso el Proyecto "Filosofía con niños" como una experiencia piloto en la escuela primaria o actual EGB. Las diferencias con respecto a la primera escuela son notorias dado que en este caso la filosofía no forma parte de una estructura curricular sino que se encuentra en una etapa de prueba. ¿Por qué se propuso hacer filosofía con niños? ¿Cómo surge esta propuesta? deberían ser los primeros interrogantes que intentemos responder. Para esto, tengo que mencionar inevitablemente parte de mi historia personal puesto que soy egresada de esta escuela y eso no sería significativo sino hubiera sentido lo valioso que podría haber sido tener en esos años de estudiante la oportunidad de hacer filosofía, es decir, de asumir una actitud reflexiva ante las cosas. Teniendo en cuenta, además, las ventajas producidas en la escuela donde ya se venía trabajando con filosofía, sugerí implementar el proyecto. El paso hacia la experiencia concreta y tangible de dicho plan llevó su tiempo, como suele ocurrir con la aparición de algo nuevo. Fueron diversas las reacciones de los miembros pertenecientes a la Institución, el consejo escolar, por ejemplo, conformado por algunos padres, se mostró muy entusiasmado y aprobó el proyecto casi inmediatamente. En cambio, los directivos miraban con desconfianza y demoraban en tomar una decisión. Cuando finalmente se dio inicio a esta experiencia, eran los niños y los docentes quienes estaban sobresaltados. "¿Qué es filosofía?" preguntaban los chicos "¿Tiene algo que ver con la piedra filosofal? ¿Qué vamos a hacer en filosofía? ¿Nos van a poner nota en la libreta? ¿Por qué ahora tenemos filosofía y antes no?". Preguntas que eran esperadas, teniendo en cuenta lo extraño que puede resultar esta palabra, sobre todo en un ámbito donde estuvo siempre ausente. Preguntas que actualmente los niños les responden a sus padres y a sus hermanos, y que han dejado de representar sentimientos de temor y amenaza ante lo desconocido. En el transcurso de la experiencia, las resistencias se fueron apaciguando y los docentes, directivos y niños se mostraron altamente entusiasmados. En algunos momentos, incluso prevalecía cierta idealización de lo que se esperaba que logre esta experiencia, se pensaba que hacer filosofía con niños constituía una garantía para resolver problemas de índole socio cultural como pueden ser la intolerancia o la discriminación.

Lo que me interesa resaltar del trabajo en esta escuela es la actitud sumamente participativa que asumieron los niños, quienes se han encontrado por primera vez con la oportunidad de ser ellos los que tomen mayores decisiones en el manejo del discurso, (cuestión que considero significativa si tenemos en cuenta que el poder de la palabra en una escuela está por lo general a cargo de los adultos. No dejo de percibirme a mi misma como parte de este juego de poder, pero tampoco dejo de ver la posibilidad de modificar las reglas de dicho juego). La participación a la que hago referencia tiene que ver también con el estrecho vínculo que se establece entre la vida y la reflexión, pues no hay otra manera de pensar que no sea desde la propia existencia, lo que representa un modo de superar la constante disociación que experimentan los niños entre el mundo de la escuela y el mundo de la vida. La experiencia es, aún incipiente, pero movilizante, no sólo por los resultados que se han mencionado sino sobre todo por la sensación de que al salir del aula, algo ha cambiado, algo ha dejado de ser lo que era, posiblemente ocurra lo que decía Neruda, que "nosotros, los de entonces, ya no somos los mismos". 


\section{FINAL ABIERTO}

Hemos señalado dos modos de entender la filosofía correspondientes a su vez con dos miradas acerca de la infancia. En una posición que concibe la filosofía como un saber conceptual ya delimitado o definido y en una postura que niega la posibilidad de búsqueda propia de la infancia, no resulta plausible hacer filosofía con niños. Si entendemos, en cambio, la filosofía como un modo particular de existir y vincularse con el mundo, desde una actitud sobre todo interrogativa y si aceptamos realmente la capacidad de cuestionar que tienen los niños, es posible, entonces, pensar en un punto de conexión entre ambos.

Dadas estas condiciones, podremos involucrarnos de un modo más auténtico en el pensar, siendo concientes que aquel que busca o pregunta no conoce las respuestas de antemano, no sabe en definitiva, cuál es el final del juego.

\section{BIBLIOGRAFÍA}

WAKSMAN, Vera; KOHAN, Walter. Filosofía con niños: Aportes para el trabajo en clase. Bs As: Novedades Educativas, 2000.

KOHAN, Walter; WAKSMAN, Vera. (comp). Filosofía para niños: Discusiones y propuestas. Bs As: Novedades Educativas, 2000.

SCHKOLNIK, Samuel. Desafíos a la razón: La filosofía y el NOA. Santiago del Estero: UNSE, 1999.

SANTIAGO, Gustavo. Filosofía con los más pequeños: Fundamentos y experiencias. Bs As: Novedades Educativas, 2002.

(1) Docente de filosofía en el nivel inicial y EGB 1 y 2 en el Instituto "Herman Hollerith" y en la Escuela Integral Argentina Hebrea "Independencia". Dirección de correo electrónico: julteitel@hotmail.com 